\title{
Reação da densidade e das propriedades mecânicas de três espécies amazônicas submetidas a ensaio de campo
}

\author{
Danyelle Santos Ribeiro, Alexandre Leão Gonçalves, Cleomar Ferreira Melo, \\ Alisson Rodrigo Souza Reis*
}

Faculdade de Engenharia Florestal, Universidade Federal do Pará, Altamira-PA, Brasil.

\begin{abstract}
RESUMO Este trabalho teve como objetivo avaliar a resistência natural, a densidade e as propriedades mecânicas de três espécies amazônicas, utilizadas na construção civil, quando submetidas a ensaio de campo. Para tanto, foram utilizados 36 corpos de prova das espécies Bagassa guianensis (tatajuba), Dinizia excelsa (angelim vermelho) e Vochysia maxima (guaruba), com dimensões de $2 \times 2 \times 30 \mathrm{~cm}$ (radial, tangencial e longitudinal), em dois ambientes de apodrecimento. No período de oito meses, os corpos de prova foram periodicamente pesados e avaliados por um critério de notas para determinação do índice de deterioração. Posteriormente, os corpos de prova foram submetidos à ensaios destrutivos, onde foram determinadas, as cargas de compressão normal, compressão paralela e flexão estática. A madeira de guaruba apresentou a menor resistência natural considerando os valores apresentados do índice de deterioração e análise das variáveis. A aplicação do teste mecânico mostrouse eficaz para determinar a espécie mais apropriada na utilização de peças estruturais, contudo estudos adicionais devem ser realizados para confirmação.

Palavras-chave: biodeterioração; madeiras amazônicas; resistência mecânica.
\end{abstract}

\section{Reaction of the density and mechanical properties of three amazon species submitted to field testing}

\begin{abstract}
The objective of this work was to evaluate the behavior of three amazonian species used in construction, being submitted to the field test, with a natural resistance and mechanical properties. Thirty specimens of the species, Bagassa guianensis (tatajuba), Dinizia excelsa (angelim rouge) and Vochysia maxima (guaruba) were used, with dimensions of 2x2x30 $\mathrm{cm}$, radial, tangential and longitudinal, respectively, in two environments. In the period of eight months, as samples were periodically weighed and evaluated by a criterion of notes to determine the deterioration index. Additionally, as samples were submitted to destructive tests, where are the tests of normal compression, parallel compression and static bending. The guaruba wood presents a lower natural resistance considering the values of the index of deterioration and analysis of the variables. The application of the mechanical test proved to be effective to determine a more appropriate species in the use of structural parts. Keywords: biodeterioration; mechanical strength; field test.
\end{abstract}

\section{Introdução}

O setor madeireiro e a construção civil somam parcela significativa na economia nacional por meio do uso da madeira, que é um material com propriedades únicas e atraentes, com diversas aplicações, o que a faz ser utilizada em grande escala, como na construção de casa, artesanato e marchetaria (CAPANEMA et al., 2013). O setor construtivo é um dos grandes consumidores dos produtos madeireiros, assim práticas como a reutilização, o uso adequado e até mesmo a substituição por outros produtos, devem ser usadas para minimizar o consumo maciço desse produto no setor e diminuir os impactos e custos (CAPANEMA et al., 2013).

Segundo o Instituto brasileiro de geografia e estatística (IBGE 2015), o Brasil, no ano de 2015, contribuiu com R\$ 
18,5 bilhões na produção primária florestal na economia nacional, com destaque para produtos da silvicultura, representando $74,3 \%$ e da extração vegetal com $25,3 \%$. O comércio mundial de madeira, de modo geral, vem sofrendo com a carência e a oferta limitada de produtos madeireiros nos últimos anos (OIMT, 2014). O mercado consumidor de madeira em Altamira-PA vem utilizando espécies com características distintas que são empregadas na construção civil. Entre essas espécies estão o Angelim Vermelho (Dinizia excelsa Ducke), Guaruba (Vochysia maxima Ducke) e a Tatajuba (Bagassa guianensis Aubl.), bastantes utilizadas no mercado madeireiro amazônico.

Segundo Zenid (2009), a madeira dispõe de diversas propriedades que a tornam atraente frente a outros materiais. Dentre elas, são normalmente apontadas a alta resistência específica, baixo consumo energético para seu processamento, ótimo isolante térmico e elétrico, além de fácil trabalhabilidade, tanto por máquinas como manualmente.

Ao empregarmos a madeira na construção civil é imprescindível determinar seu comportamento como elemento estrutural, a sua classificação e a caracterização das propriedades físicas e mecânicas, quando da sua utilização em peças estruturais (ALMEIDA et al., 2013). Contudo, as madeiras amazônicas, historicamente, são consideradas altamente resistentes, mesmo sem a confirmação por ensaios mecânicos. Aliado a isso, o uso indiscriminado dessas madeiras pode causar problemas estruturais nas construções, em virtude do desconhecimento das suas características anatômicas, físicas e mecânicas, resultando em desperdício de material.

Segundo Bradford et al. (2014), um dos fatores que pode interferir no comportamento da madeira em ensaio de campo é o clima. As mudanças climáticas fazem com que alguns agentes deterioradores, a exemplo os fungos ou cupins, diminuam, ou até mesmo cessem, as suas atividades, principalmente devido à variação da temperatura média e disponibilidade de umidade. Fato corroborado por Trevisan et al, (2007), quando afirmaram que variações ambientais, especialmente com relação à temperatura e umidade, têm importância fundamental no desenvolvimento dos microrganismos aptos ao ataque e na agilidade de decomposição da madeira.

Nos últimos anos, diversos estudos na área de resistência natural, obtidos por meio de ensaios de campo e resistência mecânica com espécies amazônicas vem sendo desenvolvidos por inúmeros autores, como em trabalhos de Melo et al. (2010), Corassa et al. (2013), Almeida et al. (2013), Marcondes et al. (2013), Ribeiro et al. (2014), Stangerlin et al. (2015) e Pilocelli et al. (2015). Diante disso, o objetivo da pesquisa consistiu em avaliar o comportamento de três espécies amazônicas amplamente utilizadas na construção civil, entre as quais estão o angelim vermelho, Guaruba e Tatajuba. O material foi submetido ao ensaio de campo, em duas situações distintas, com ênfase na resistência natural e nas propriedades mecânicas.

\section{Material e Métodos}

\section{Caracterização da área de estudo}

Os ensaios de campo foram realizados no período de setembro de 2015 a maio de 2016, em dois ambientes: campo aberto (área livre de vegetação), coordenadas S $03^{\circ} 11.860^{\prime} \mathrm{e}$ W $052^{\circ} 11.344^{\prime}$ com elevação de 156 m, e campo fechado (área de floresta nativa), coordenadas S $03^{\circ} 11.890^{\prime}$ e W $052^{\circ} 23.205^{\prime}$ com elevação de 145 m, ambos no município de Altamira-PA. Segundo a classificação de Köppen, o clima do município classificou-se como equatorial Am e Aw. 


\section{Coleta e preparo dos corpos de prova}

As madeiras foram coletadas em duas serrarias, sendo uma no município de Altamira/PA e outra em Anapu/PA. Foram utilizadas três espécies: Angelim Vermelho (Dinizia excelsa Ducke), Guaruba (Vochysia maxima Ducke) e Tatajuba (Bagassa guianensis Aubl.). Os corpos de prova foram confeccionados - com as dimensões nominais de 2,0 x $2,0 \times 30,0 \mathrm{~cm}$ (radial, tangencial e longitudinal, respectivamente), com adaptação das normas técnicas - NBR 7190 da associação brasileira de normas técnicas, sendo utilizados nos ensaios de deterioração à campo e posteriormente, submetidos aos ensaios mecânicos.

Foram selecionados 36 corpos de prova por espécie, totalizando 108 corpos de prova, que foram submetidos ao condicionamento, secos em estufa a $100^{\circ} \mathrm{C}$, até atingirem peso constante. Posteriormente, foram medidos e pesados com o auxílio de paquímetro digital de 0,01mm e balança analítica de 0,001g, respectivamente, de acordo com metodologia descrita por Pilocelli et al. (2015).

\section{Instalação e avaliação dos ensaios de}

\section{deterioração}

Os corpos de prova, em cada ambiente de deterioração (campo aberto e floresta), foram distribuídos em três blocos casualizados, totalizando 18 corpos de prova para cada espécie, em cada ambiente, conforme a Figura 1. Os mesmos foram enterrados, parcialmente, ou seja, $15 \mathrm{~cm}$ do seu comprimento, distantes $5 \mathrm{~cm}$ um do outro. A distância entre os blocos foi padronizada em $50 \mathrm{~cm}$.

A cada 60 dias, durante seis meses, foram retirados quatro corpos de prova por espécie em cada ambiente de deterioração, de modo a monitorar parcialmente a durabilidade natural das madeiras. A distinção final da durabilidade natural foi realizada aos oito meses de instalação dos ensaios de campo, sendo amostrados seis corpos de prova para cada espécie em cada ambiente de deterioração.

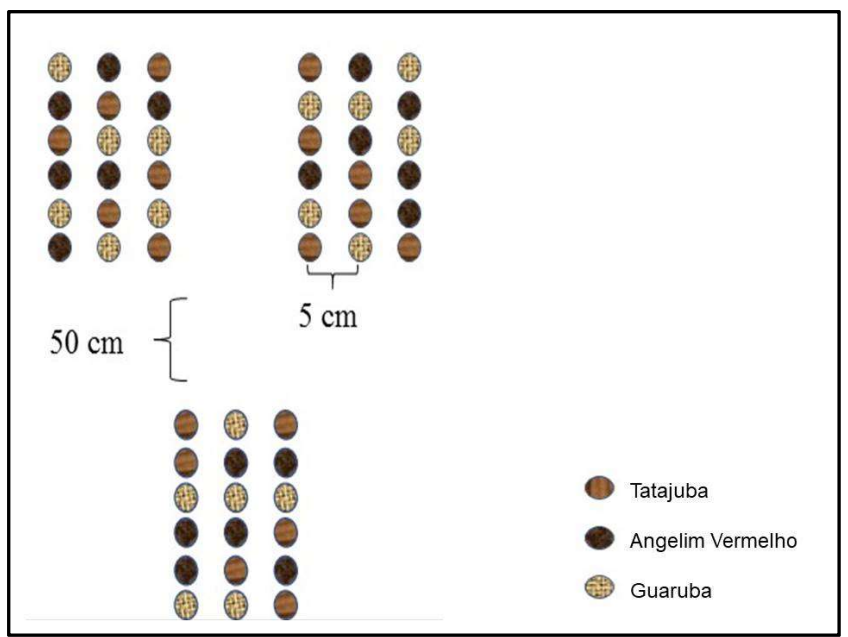

Figura 1. Croqui com disposição do experimento.

Figure 1. Sketch with layout of the experiment.

A avaliação da resistência natural da madeira procedeu por meio de verificação visual da parte dos corpos de prova que estavam em contato com o solo, adotando-se o índice de deterioração proposto por Lepage (1970), o qual recomenda tal avaliação pelo critério subjetivo de notas. O índice de comportamento foi admitido para estabelecer o estado médio de deterioração de cada amostra, como descrito na Tabela 1.

\section{Ensaios mecânicos}

Para condução dos ensaios mecânicos, foram utilizados os corpos de prova utilizados no ensaio de campo, com a finalidade de avaliar o grau de resistência nos ambientes e forma que os corpos de provas foram submetidos. Os ensaios mecânicos foram realizados de acordo com a NBR 7190 (ABNT, 1997) - e executados no Laboratório de Engenharia Civil, da Universidade Federal do Pará, Campus Tucuruí. Foram realizados os testes de densidade básica (Equação 1), compressão paralela às fibras (Equação 2), compressão normal às fibras (Equação 3) e flexão (Equação 4). 
Tabela 1. Classificação do índice de deterioração da madeira. (Fonte: Lepage, 1970)

Table 1. Classification of wood deterioration index.

\begin{tabular}{lll}
\hline Estado de Sanidade Nota & Índice de comportamento
\end{tabular}

\begin{tabular}{lcc}
\hline Sadio - Nenhum ataque & 0 & 100 \\
\hline Ataque leve ou superficial de fungos ou térmitas & 1 & 90
\end{tabular}

\begin{tabular}{lll}
\hline Ataque evidente, mas moderado de fungos ou térmitas & 2 & 70
\end{tabular}

\begin{tabular}{lll}
\hline Apodrecimento intenso ou ataque interno de térmitas & 3 & 40
\end{tabular}

Quebra - perda quase total de resistência

4

0

Pbas $=\frac{\mathrm{ms}}{\mathrm{Vsat}} \quad$ (Equação 1)

Onde: $\mathrm{ms}$ : massa seca $(\mathrm{kg})$; Vsat: volume saturado $\left(\mathrm{m}^{3}\right)$.

A compressão paralela às fibras:

$\mathrm{fc} 0=\frac{\text { fc0, máx. }}{\mathrm{A}} \quad$ (Equação 2)

Onde: fc0, máx. é a máxima força de compressão $(\mathrm{N})$; A: área inicial $\left(\mathrm{m}^{2}\right)$.

A compressão normal às fibras:

fc90 $=\frac{\sigma 50 \%-\sigma 10 \%}{\varepsilon 50 \%-\varepsilon 10 \%} \quad$ (Equação 3)

Onde: $\sigma 10 \%$ e $\sigma 50 \%$ são as tensões de compressão normal correspondentes a $10 \%$ e $50 \%$ da resistência convencional e, $\mathrm{f}$ c90; $\varepsilon 10 \%$ e $\varepsilon 50 \%$ : são as deformações específicas medidas na direção normal às fibras correspondentes às tensões $\sigma 10 \% \mathrm{e}$ $\sigma 50 \%$.

E a Flexão:

$\mathrm{fM}=\frac{\text { Mmáx. }}{\text { We }} \quad$ (Equação 4$)$

Onde: Mmáx. é o máximo momento aplicado ao corpo-deprova, em newtons-metro; We é o módulo de resistência elástico da seção transversal do corpo-de-prova, dado por bh2/6, em metros cúbicos.

\section{Análise estatística}

Para a análise multivariada foi utilizada a análise de componentes principais, utilizando como fatores a variação das dimensões na secção tangencial, radial e o peso dos corpos de prova, a nível de 5\% de probabilidade de erro. Para os testes mecânicos utilizou-se teste $\mathrm{F}$ com a mesma significância e quando observada significância aplicou-se o teste Tukey a $5 \%$ de probabilidade, com auxílio do programa estatístico Assistat 7.7 e pacote estatístico R Core.

\section{Resultados e Discussão}

\section{Guaruba}

A madeira de Guaruba apresentou densidade básica de 0,54 g.cm $\mathrm{cm}^{3}$ nos corpos de prova testemunhas, para essa propriedade Nahuz (2013) encontrou o valor de 0,49 g.cm ${ }^{3}$ e ainda caracterizou a espécie como suscetível a moderadamente resistente ao ataque de cupins e fungos, enquanto que Vale et al. (2009) determinaram a densidade para a espécie Vochysia thyrsoidea de $0,55 \mathrm{~g} . \mathrm{cm}^{3}$. Segundo Jati et al (2014), Silva et al $(2015 ; 2016)$ o conhecimento da densidade da madeira pode-se verificar as propriedades funcionais e esta pode sofrer influencias bióticas e abióticas.

Ao analisar o estado de deterioração dos corpos de prova, aplicando-se o índice de sanidade, foi constatado que apenas $5,56 \%$ dos corpos de prova sofreram ataques leves de fungos e térmitas, compreendendo um índice de comportamento de 
90, não comprometendo visualmente a amostra analisada, e $94,44 \%$ não sofreram nenhum ataque, sendo atribuído o índice de 100. Para determinar o grau de deterioração dos corpos de prova, foram analisadas estatisticamente todas as variáveis. Nas seções radial e tangencial, tanto em campo aberto quanto em campo fechado, não houveram diferenças, conforme descritos na Tabela 2.

E com relação ao peso, as variações nos corpos de prova em campo aberto ocorreram do segundo ao oitavo mês do experimento, diferindo apenas da testemunha, que foram os corpos de prova antes de irem a campo, com teor de umidade a $12 \%$. Observou-se uma diminuição do peso no oitavo mês, em virtude, do experimento ter sido encerrado no período mais seco da região amazônica. Já nos corpos de prova que estavam em uma área de floresta nativa, a variação do peso foi aumentando constantemente, pois em áreas fechadas as madeiras tendem a adquirir mais umidade, devido ser limitada a entrada de radiação e vento, proporcionando o aumento de massa.

Para confirmar a resistência natural da madeira, a Tabela 3 mostra que a espécie Vochysia maxima, apresentou variação apenas no teste de compressão paralela, onde a média foi de 18,56 MPa. Essa variação pode ser explicada pelo fato de a madeira ser considerada de densidade baixa, por possuir fibras de tamanho médio e parede fina (REIS et al 2015; IAWA, 1989) refletindo assim na resistência. O comportamento nesse teste foi diferente ao encontrado por Nahuz (2013), os quais obtiveram resultados de 29,04 MPa para a madeira verde e $47,6 \mathrm{MPa}$ para a madeira a $12 \%$ de umidade para a mesma espécie.

Tabela 2. Análise da variância das dimensões das seções radiais, tangenciais e o peso para a madeira de Guaruba (Vochysia maxima).

Table 2. Analysis of the variance of the radial, tangential and weight of Guaruba (Vochysia maxima).

\begin{tabular}{|c|c|c|c|c|c|}
\hline \multicolumn{6}{|c|}{ Guaruba } \\
\hline \multicolumn{6}{|c|}{ Campo aberto } \\
\hline \multirow{3}{*}{ Coleta } & \multicolumn{5}{|c|}{ Média da variação } \\
\hline & \multicolumn{2}{|c|}{ Radial } & \multicolumn{2}{|c|}{ Tangencial } & \multirow{2}{*}{ Peso $(g)$} \\
\hline & Exposto & Não exposto & Exposto & Não exposto & \\
\hline Testemunha & $2,04 \mathrm{a}$ & $2,02 \mathrm{a}$ & $2,03 \mathrm{a}$ & $1,99 \mathrm{a}$ & $71,16 \mathrm{~b}$ \\
\hline 2 meses & $2,02 \mathrm{a}$ & $2,07 \mathrm{a}$ & $2,00 \mathrm{a}$ & $2,04 \mathrm{a}$ & $86,92 \mathrm{a}$ \\
\hline 4 meses & $1,99 \mathrm{a}$ & $2,24 \mathrm{a}$ & $2,00 \mathrm{a}$ & $2,03 \mathrm{a}$ & $85,03 \mathrm{a}$ \\
\hline 6 meses & $2,05 \mathrm{a}$ & $2,05 \mathrm{a}$ & $2,07 \mathrm{a}$ & $2,08 \mathrm{a}$ & $90,97 \mathrm{a}$ \\
\hline 8 meses & $2,07 \mathrm{a}$ & $2,11 \mathrm{a}$ & $2,06 \mathrm{a}$ & $2,09 \mathrm{a}$ & $86,88 \mathrm{a}$ \\
\hline \multicolumn{6}{|c|}{ Campo fechado } \\
\hline \multirow{3}{*}{ Coleta } & \multicolumn{5}{|c|}{ Média da variação } \\
\hline & \multicolumn{2}{|c|}{ Radial } & \multicolumn{2}{|c|}{ Tangencial } & \multirow{2}{*}{ Peso $(g)$} \\
\hline & Exposto & Não exposto & Exposto & Não exposto & \\
\hline Testemunha & $2,04 \mathrm{a}$ & $2,00 \mathrm{a}$ & $2,06 \mathrm{a}$ & $2,02 \mathrm{a}$ & $70,99 \mathrm{~d}$ \\
\hline 2 meses & $2,02 \mathrm{a}$ & $1,97 \mathrm{a}$ & $2,05 \mathrm{a}$ & $2,02 \mathrm{a}$ & $82,06 \mathrm{c}$ \\
\hline 4 meses & $2,02 \mathrm{a}$ & $1,99 \mathrm{a}$ & $2,13 \mathrm{a}$ & $2,08 \mathrm{a}$ & $82,86 \mathrm{c}$ \\
\hline 6 meses & $2,07 \mathrm{a}$ & $2,03 \mathrm{a}$ & $2,13 \mathrm{a}$ & $2,05 \mathrm{a}$ & $99,47 \mathrm{~b}$ \\
\hline 8 meses & $2,13 \mathrm{a}$ & $2,12 \mathrm{a}$ & $2,19 \mathrm{a}$ & $2,21 \mathrm{a}$ & $114,17 \mathrm{a}$ \\
\hline
\end{tabular}

As médias seguidas pela mesma letra na mesma coluna, não diferem estatisticamente entre si. Foi aplicado o Teste de Tukey ao nível de $5 \%$ de probabilidade. 
Tabela 3. Análise da variância para o teste mecânico na madeira de guaruba.

Table 3. Analysis of variance for the mechanical test on guaruba wood.

\begin{tabular}{c|c|c|c}
\hline \multirow{2}{*}{ Coleta } & \multicolumn{3}{|c}{ Muaruba } \\
\cline { 2 - 5 } & Compressão Normal & Compressão Paralela & Flexão \\
\hline 2 meses & $12,61^{ \pm 31.47^{*}} \mathrm{a}$ & $0,79^{ \pm 0.23} \mathrm{~b}$ & $0,10^{ \pm 0.01} \mathrm{a}$ \\
\hline 4 meses & $13,06^{ \pm 0.07} \mathrm{a}$ & $0,76^{ \pm 0.07} \mathrm{~b}$ & $0,09^{ \pm 0.02} \mathrm{a}$ \\
\hline 6 meses & $11,65^{ \pm 0.04} \mathrm{a}$ & $0,62^{ \pm 0.03} \mathrm{~b}$ & $0,07^{ \pm 0.00} \mathrm{a}$ \\
\hline 8 meses & $18,64^{ \pm 26.36} \mathrm{a}$ & $18,56^{ \pm 5.46} \mathrm{a}$ & $0,0^{ \pm 0.00} \mathrm{a}$ \\
\hline
\end{tabular}

As médias seguidas pela mesma letra na mesma coluna, não diferem estatisticamente entre si. Foi aplicado o

Teste de Tukey ao nível de $5 \%$ de probabilidade. ${ }^{\star}$ Desvio padrão em relação a média.

Enquanto na pesquisa conduzida por Dias; Lahr (2004), a espécie Vochysia haenkeana, apresentaram valores de $60 \mathrm{MPa}$ para a compressão paralela e $87 \mathrm{MPa}$ para a flexão e no estudo realizado por Cardoso et al. (2012) com Qualea dinizii a madeira apresentou valores aproximados (52,26 MPa para compressão e $87,85 \mathrm{MPa}$ para flexão) em todos os testes mecânicos realizados neste estudo e com base nesses fatores.

Os autores indicaram essas madeiras para a construção civil, no estabelecimento de conexões, encaixes, treliças e, principalmente, na sobreposição de peças, corroborando com os resultados encontrados neste trabalho, ressaltando que as espécies pertencem à mesma família botânica da espécie estudada.

Deve-se considerar que diversos fatores podem influenciar os testes mecânicos, sendo um deles a característica anatômica da madeira, sitio de coleta, temperatura e umidade da madeira e do ambiente. Apesar dessas diferenças pequenas de valores, pode-se inferir que as madeiras de vochysiaceae apresentam comportamento mecânico semelhantes.

\section{Angelim Vermelho}

A densidade básica da testemunha encontrada para a espécies neste trabalho foi de 0,92 g.cm³ e Araújo (2007), estudando a mesma espécie encontrou o valor de 0,83 g.cm $\mathrm{cm}^{3}$. A partir de resultados coletados em ensaios de campo, a madeira de angelim vermelho é considerada uma espécie altamente durável, com vida média maior que oito anos (JESUS et al. 1998).

No ensaio de campo, os corpos de prova de Dinizia excelsa, visualmente, não sofreram nenhum ataque de agentes deterioradores, e segundo a metodologia de Lepage (1970) é atribuído o índice de 100. Esse índice não confirma que microrganismos e processos abióticos não tenham agido na deterioração da madeira de forma intensa, sendo a sua interpretação pertinente aos danos superficiais, evidentes e macroscópicos (TREVISAN et al., 2008). Outro fator que contribui na resistência da madeira de angelim é a presença de compostos fenólicos, conforme observado por Gomes; Bandeira (1983) na madeira de Hymenolobium excelsum.

Verifica-se na Tabela 4, que para as dimensões analisadas, o angelim vermelho mostrou-se variável nas dimensões da seção radial, na parte exposta em campo aberto e não exposto em campo fechado, com menor média em dois meses de experimento de $2,03 \mathrm{~cm}$ e maior $2,17 \mathrm{~cm}$ no sexto mês do experimento (campo aberto) e em ambiente fechado de 2,11 $\mathrm{cm}$ e a testemunha com $2,22 \mathrm{~cm}$ no quarto e sexto mês do ensaio.

$\mathrm{Na}$ seção tangencial e em campo aberto, não houve variação, somente em campo fechado, onde as dimensões variaram no lado não exposto (parte enterrada). 
Tabela 4. Análise da variância das seções radiais, tangenciais e peso em campo aberto e campo fechado de Angelim Vermelho (Dinizia excelsa).

Table 4. Analysis of the variance of the radial, tangential and weight in open field and closed field of Angelim Vermelho (Dinizia excelsa).

\begin{tabular}{|c|c|c|c|c|c|}
\hline \multicolumn{6}{|c|}{ Angelim Vermelho } \\
\hline \multicolumn{6}{|c|}{ Campo aberto } \\
\hline \multirow{3}{*}{ Coleta } & \multicolumn{5}{|c|}{ Média da variação (cm) } \\
\hline & \multicolumn{2}{|c|}{ Radial } & \multicolumn{2}{|c|}{ Tangencial } & \multirow{2}{*}{ Peso $(g)$} \\
\hline & Exposto & Não exposto & Exposto & Não exposto & \\
\hline Testemunha & $2,07 \mathrm{ab}$ & $2,08 \mathrm{a}$ & $2,03 \mathrm{a}$ & $2,05 \mathrm{a}$ & $127,07 \mathrm{~b}$ \\
\hline 2 meses & $2,03 \mathrm{~b}$ & $2,11 \mathrm{a}$ & $2,03 \mathrm{a}$ & $2,18 \mathrm{a}$ & $143,14 \mathrm{ab}$ \\
\hline 4 meses & $2,11 \mathrm{ab}$ & $2,11 \mathrm{a}$ & $2,05 \mathrm{a}$ & $2,10 \mathrm{a}$ & $145,42 \mathrm{a}$ \\
\hline 6 meses & $2,17 \mathrm{a}$ & $2,12 \mathrm{a}$ & $2,09 \mathrm{a}$ & $2,13 \mathrm{a}$ & $158,01 \mathrm{a}$ \\
\hline 8 meses & $2,07 \mathrm{ab}$ & $2,19 \mathrm{a}$ & $2,12 \mathrm{a}$ & $2,17 \mathrm{a}$ & $158.07 \mathrm{a}$ \\
\hline \multicolumn{6}{|c|}{ Campo fechado } \\
\hline \multirow{3}{*}{ Coleta } & \multicolumn{5}{|c|}{ Média da variação $(\mathrm{cm})$} \\
\hline & \multicolumn{2}{|c|}{ Radial } & \multicolumn{2}{|c|}{ Tangencial } & \multirow{2}{*}{ Peso $(g)$} \\
\hline & Exposto & Não exposto & Exposto & Não exposto & \\
\hline Testemunha & $2,05 \mathrm{a}$ & $2,11 \mathrm{~b}$ & $2,03 \mathrm{a}$ & $2,06 \mathrm{~b}$ & $126,23 \mathrm{~b}$ \\
\hline 2 meses & $2,01 \mathrm{a}$ & $2,15 \mathrm{ab}$ & $2,10 \mathrm{a}$ & $2,14 \mathrm{a}$ & $153,58 \mathrm{a}$ \\
\hline 4 meses & $2,13 \mathrm{a}$ & $2,22 \mathrm{a}$ & $2,09 \mathrm{a}$ & $2,12 \mathrm{ab}$ & $159,97 \mathrm{a}$ \\
\hline 6 meses & $2,16 \mathrm{a}$ & $2,22 \mathrm{a}$ & $2,08 \mathrm{a}$ & $2,17 \mathrm{a}$ & $161,53 \mathrm{a}$ \\
\hline 8 meses & $2,08 \mathrm{a}$ & $2,14 \mathrm{ab}$ & $2,10 \mathrm{a}$ & $2,13 \mathrm{ab}$ & $160,63 \mathrm{a}$ \\
\hline
\end{tabular}

As médias seguidas pela mesma letra na mesma coluna, não diferem estatisticamente entre si. Foi aplicado o Teste de Tukey ao nível de $5 \%$ de probabilidade.

Observa-se que os corpos de provas foram aumentando gradativamente o peso, em ambos os ambientes, virtude da absorção da umidade. Isso ocorre porque a região amazônica, por estar próxima da linha do equador, não possui estações do ano definidas e com isso possui instabilidade no clima e índice pluviométrico. Esse comportamento de aumento gradativo foi observado em duas espécies, somente a quaruba apresentou comportamento diferente.

Para confirmar os ensaios de campo, foram realizados testes mecânicos, onde os corpos de prova também se mostraram estáveis aos testes realizados, variando após o oitavo mês na compressão paralela com média de $40.99 \mathrm{MPa}$ e flexão com $0.45 \mathrm{MPa}$, como descritos na Tabela 5.
Os resultados encontrados foram diferentes dos encontrados por Araújo (2007), que estudando as propriedades físicas e mecânicas de espécies tropicais, encontrou resultados de 156,91 MPa para a flexão, $85,61 \mathrm{MPa}$ na compressão paralela e 14, $81 \mathrm{MPa}$ na compressão normal, estudando a mesma espécie.

Para a espécie da mesma família, Pterodon pubescens, Vale et al. (2011), encontraram valor médio de menor resistência à compressão paralela de $72 \mathrm{Mpa}$, variando de 69 a $75 \mathrm{Mpa}$, enquanto que a média encontrada para a resistência à compressão normal foi de $22 \mathrm{MPa}$, variando de 19 a $28 \mathrm{MPa}$. Dias; Lahr (2004), encontraram uma média para à compressão paralela de $78 \mathrm{MPa}$, e para a flexão estática de 110 MPa, para a mesma espécie. 
Tabela 5. Análise da variância para os ensaios mecânicos na madeira de angelim vermelho.

Table 5. Analysis of the variance for the mechanical test on red angelim wood.

\begin{tabular}{c|c|c|c}
\hline \multirow{2}{*}{ Coleta } & \multicolumn{3}{|c}{ Angelim Vermelho } \\
\cline { 2 - 4 } & Compressão Normal & Códia de variação (MPa) & Flexão \\
\hline \multirow{2}{*}{2 meses } & $55,92^{ \pm 32.45^{*}} \mathrm{a}$ & $1,16^{ \pm 0.21} \mathrm{~b}$ & $0,23^{ \pm 0.02} \mathrm{~b}$ \\
\hline 4 meses & $58,32^{ \pm 0.26} \mathrm{a}$ & $1,23^{ \pm 0.19} \mathrm{~b}$ & $0,10^{ \pm 0.01} \mathrm{~b}$ \\
\hline 6 meses & $55,80^{ \pm 0.15} \mathrm{a}$ & $0,88^{ \pm 0.04} \mathrm{~b}$ & $0,11^{ \pm 0.04} \mathrm{~b}$ \\
\hline 8 meses & $61,31^{ \pm 16.55} \mathrm{a}$ & $40,99^{ \pm 5.35} \mathrm{a}$ & $0,45^{ \pm 0.08} \mathrm{a}$ \\
\hline
\end{tabular}

As médias seguidas pela mesma letra na mesma coluna, não diferem estatisticamente entre si. Foi aplicado o Teste de Tukey ao nível de $5 \%$ de probabilidade.* Desvio padrão em relação à média

\section{Tatajuba}

A densidade básica da madeira de Bagassa guianensis foi de 0,85 g. $\mathrm{cm}^{3}$, diferindo-se dos resultados encontrados por Corassa et al. (2013) e Araújo (2007), sendo de 0,70 g.cm³ , e o Nahuz (2013) com densidade de 0,68 g.cm ${ }^{3}$.

$\mathrm{Na}$ atribuição de notas pelo índice de comportamento, a espécie sofreu ataque leve de fungos e térmitas em apenas um dos corpos de prova ensaiadas, apresentando um índice de comportamento de 90 , não afetando visualmente o corpo de prova analisado.

Vale observar que a presença de extrativos na madeira pode proporcionar maior ou menor resistência à espécie florestal, assim, para uma conclusão mais segura, sugere-se a realização de análise dos extrativos das madeiras em estudo (CORASSA et al., 2014).

Segundo Carvalho; Carvalho (1993), a madeira da tatajuba apresenta considerável durabilidade em relação à resistência ao ataque de fungos, tem baixa resistência a períodos chuvosos e com alta umidade, fendas aparecem quando a madeira fica exposta ao sol sem nenhuma cobertura de proteção, sendo classificada como de fácil preservação.

Com base nos dados da Tabela 6, não foi detectada diferença significativa entre os ambientes de deterioração, tanto para a variável radial como tangencial, o que indica que o ambiente não teve influência na deterioração da espécie.
Já para a variável peso, houve uma variação em oito meses de experimento nos dois ambientes, com média de 200,78 g e 208,65 g, respectivamente.

Observa-se que não houve diferença estatística para a compressão normal. A tensão máxima na compressão paralela, ocorreu com os corpos de prova coletados no oitavo mês, com 54,05 MPa e na flexão foi de 0.46 MPa. Dias; Lahr (2004) obtiveram resultados de $79 \mathrm{MPa}$ para a compressão normal e $110 \mathrm{MPa}$ para a flexão estática.

\section{Análise dos componentes principais - PCA}

Por meio da análise dos componentes principais, observou-se que as três espécies formaram três grupos bem delimitados. Apesar dos grupos bem destacados, as características das variações do angelim vermelho e da guaruba são um pouco semelhantes, diferenciando-se totalmente da tatajuba, isso para as variáveis, radial, tangencial e peso.

Levando em consideração a análise estatística, a Figura 2 explica $84 \%$ das variações dos dados. Nela reafirma que a espécie tatajuba não sofreu variação ao longo do tempo. O que pode ser explicado pelo fato da espécie ser bastante resistente a agentes deteriorados e ao ambiente. Já a espécie guaruba, foi a que apresentou pouca variação para as variáveis analisadas, mas por ser de média densidade é considerada a 
Tabela 6. Análise da variância das seções radiais, tangenciais e peso em campo aberto e campo fechado de Tatajuba (Bagassa guianensis).

Table 6. Analysis of the variance of the radial, tangential and weight in open field and closed field of Tatajuba (Bagassa guianensis).

Tatajuba

Campo aberto

\begin{tabular}{|c|c|c|c|c|c|}
\hline \multirow{3}{*}{ Coleta } & \multicolumn{5}{|c|}{ Média da variação $(\mathrm{cm})$} \\
\hline & \multicolumn{2}{|c|}{ Radial } & \multicolumn{2}{|c|}{ Tangencial } & \multirow{2}{*}{ Peso (g) } \\
\hline & Exposto & Não exposto & Exposto & Não exposto & \\
\hline Testemunha & $2,25 \mathrm{a}$ & $2,27 \mathrm{a}$ & $2,76 \mathrm{a}$ & $2,77 \mathrm{a}$ & $163,96 \mathrm{~b}$ \\
\hline 2 meses & $2,21 \mathrm{a}$ & $2,28 \mathrm{a}$ & $2,77 \mathrm{a}$ & $2,89 \mathrm{a}$ & $184,46 \mathrm{a}$ \\
\hline 4 meses & $2,22 \mathrm{a}$ & $2,29 \mathrm{a}$ & $2,76 \mathrm{a}$ & $2,72 \mathrm{a}$ & $180,09 \mathrm{ab}$ \\
\hline 6 meses & $2,29 \mathrm{a}$ & $2,30 \mathrm{a}$ & $2,71 \mathrm{a}$ & $2,75 \mathrm{a}$ & $196,68 \mathrm{a}$ \\
\hline 8 meses & $2,28 \mathrm{a}$ & $2,30 \mathrm{a}$ & $2,74 \mathrm{a}$ & $2,80 \mathrm{a}$ & $200,78 \mathrm{a}$ \\
\hline
\end{tabular}

\begin{tabular}{|c|c|c|c|c|c|}
\hline \multicolumn{6}{|c|}{ Campo fechado } \\
\hline \multirow{3}{*}{ Coleta } & \multicolumn{5}{|c|}{ Média da variação $(\mathrm{cm})$} \\
\hline & \multicolumn{2}{|c|}{ Radial } & \multicolumn{2}{|c|}{ Tangencial } & \multirow{2}{*}{ Peso $(g)$} \\
\hline & Exposto & Não exposto & Exposto & Não exposto & \\
\hline Testemunha & $2,27 \mathrm{a}$ & $2,27 \mathrm{a}$ & $2,75 \mathrm{a}$ & $2,80 \mathrm{a}$ & $165,13 \mathrm{c}$ \\
\hline 2 meses & $2,26 \mathrm{a}$ & $2,30 \mathrm{a}$ & $2,76 \mathrm{a}$ & $2,80 \mathrm{a}$ & $183,68 \mathrm{bc}$ \\
\hline 4 meses & $2,32 \mathrm{a}$ & $2,28 \mathrm{a}$ & $2,87 \mathrm{a}$ & $2,96 \mathrm{a}$ & $193,59 \mathrm{ab}$ \\
\hline 6 meses & $2,27 \mathrm{a}$ & $2,28 \mathrm{a}$ & $2,68 \mathrm{a}$ & $2,74 \mathrm{a}$ & $204,32 \mathrm{ab}$ \\
\hline 8 meses & $2,27 \mathrm{a}$ & $2,32 \mathrm{a}$ & $2,79 \mathrm{a}$ & $2,80 \mathrm{a}$ & $208,65 \mathrm{a}$ \\
\hline
\end{tabular}

As médias seguidas pela mesma letra na mesma coluna, não diferem estatisticamente entre si. Foi aplicado o Teste de Tukey ao nível de $5 \%$ de probabilidade.

Tabela 7. Análise da variância para o teste mecânico na madeira de tatajuba.

Table 7. Analysis of the variance for the mechanical test on tatajuba wood.

\begin{tabular}{c|c|c|c}
\hline \multirow{2}{*}{ Coleta } & \multicolumn{3}{|c}{ Tatajuba } \\
\cline { 2 - 4 } & Compressão Normal & Compressão Paralela & Flexão \\
\hline 2 meses & $48,22^{ \pm 29.55^{*}} \mathrm{a}$ & $1,66^{ \pm 0.67} \mathrm{~b}$ & $0,23^{ \pm 0.09} \mathrm{ab}$ \\
\hline 4 meses & $49,00^{ \pm 20.59} \mathrm{a}$ & $1,80^{ \pm 0.41} \mathrm{~b}$ & $0,12^{ \pm 0.007} \mathrm{~b}$ \\
\hline 6 meses & $55,81^{ \pm 0.14} \mathrm{a}$ & $1,49^{ \pm 0.21} \mathrm{~b}$ & $0,12^{ \pm 0.01} \mathrm{~b}$ \\
\hline 8 meses & $34,38^{ \pm 15.37} \mathrm{a}$ & $54,05^{ \pm 16.06} \mathrm{a}$ & $0,46^{ \pm 0.10} \mathrm{a}$ \\
\hline
\end{tabular}

As médias seguidas pela mesma letra na mesma coluna, não diferem estatisticamente entre si. Foi aplicado o Teste de Tukey ao nível de $5 \%$ de probabilidade. ${ }^{\star}$ Desvio padrão em relação a média

mais suscetível as ações do ambiente, assim como os corpos de prova de angelim vermelho que demonstraram ao longo do tempo uma variação para os componentes analisados, explicados pelo fato da espécie ser de alta densidade e o teor de umidade na madeira ter feito com que houvesse essa variação. 


\section{Conclusões}

Dentre as espécies, a madeira de guaruba apresentou o maior índice de deterioração não sendo recomendada na utilização externa. As madeiras de angelim vermelho e tatajuba por apresentarem a maior densidade e tensão máxima nos testes de compressão normal, compressão paralela e na flexão, são mais indicadas para a utilização em peças estruturais externas, por suportarem a maior carga. As madeiras sofreram inchaço em campo, porém a madeira de quaruba foi a que apresentou maior variação dimensional.

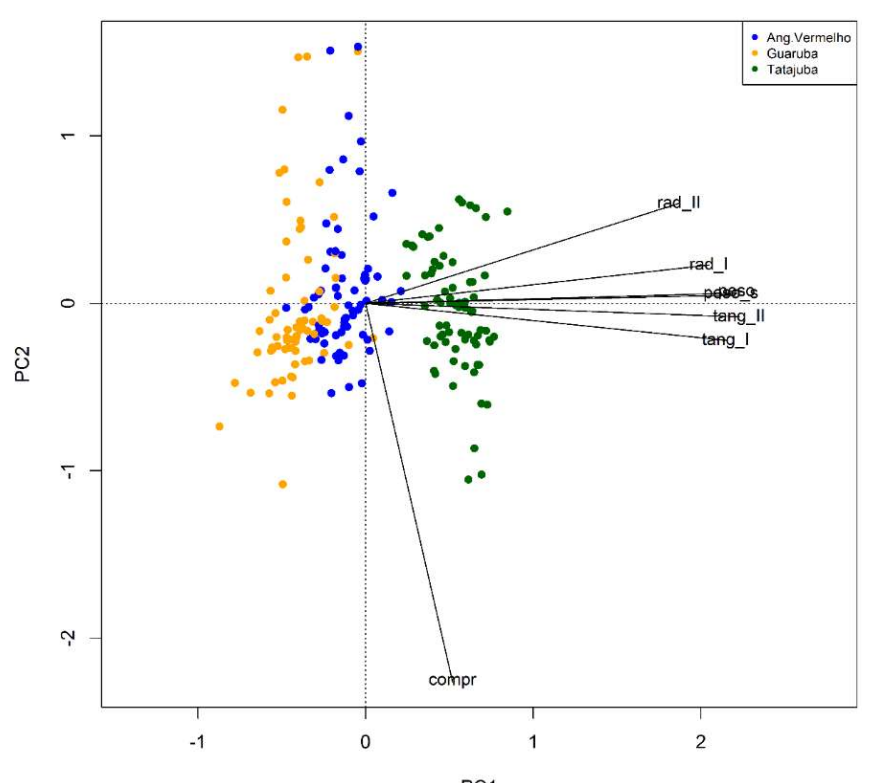

Figura 2. Disposição das espécies em relação as variáveis radial, tangencial e peso.

Figure 2. Arrangement of species in relation to radial, tangential and weight variables.

\section{Referências}

ALMEIDA, D. R.; SCALIANTE, R. M.; MACEDO, L. B.; MACÊDO, A. N.; DIAS, A. A.; CHRISTOFORO, A. L.; CALIL JUNIOR, C. Caracterização completa da madeira da espécie amazônica Paricá (Schizolobium amazonicum HERB) em peças de dimensões estruturais. Revista Árvore. ViçosaMG, v. 37, n. 6, p. 1175-1181, 2013.

ARAUJO, H. J. B. Relações funcionais entre propriedades físicas e mecânicas de madeiras tropicais brasileiras. Floresta, Curitiba, PR, n.37, p. 399-416, 2007.
ASSOCIAÇÃO BRASILEIRA DE NORMAS TÉCNICASABNT. NBR 7190:1997 Projeto de estruturas de madeira. Rio de Janeiro: ABNT, 1997. 15p.

BRADFORD, M. A.; WARREN, R. J.; BALDRIAN, P.; CROWTHER, T. W.; MAYNARD, D. S.; OLDFIELD, E. E.; WIEDER, W. R.; WOOD, S. A.; KING, J. R. Climat fails to predict wood decomposition at regional scales. Nature Climate Change. V. 4, p. 625-630, 2014.

BRASÍLIA. SERVIÇO FLORESTAL BRASILEIRO. (Org.). Panorama Econômico do Setor Florestal. Brasília: Gerência Executiva de Planejamento Florestal, 2014. 1 p. 1 v.

CAPANEMA, D. C. O.; GALVÃO, F. H.; SANTANA, K. M. F.; LUPP, L. S. L.; ALEXANDRE, R. A. S.; COSTA, T. A. R. Setor madeireiro e as consequências do uso da madeira na construção civil: Possíveis soluções. Percurso Acadêmico. Belo Horizonte. V. 3, n.6, p. 335-347, 2013.

CARdoso, C. C.; MOUTINHO, V. H. P.; MELO, L. O.; SOUSA, L. K. V. S.; SOUZA, M. R. Caracterização físicomecânica de madeiras amazônicas com aptidão tecnológica para comercialização. Revista de Ciências Agrarias. V. 55, n3, p. 176-183, 2012.

CARVALHO, M. S. P.; CARVALHO, J. O. P. de. Influência do espaçamento nas características físico-mecânicas da madeira de Bagassa guianensis Aubl. (Tatajuba) no planalto de Belterra, Pará. Comunicado Técnico, $n^{\circ} 88$, Brasília, Embrapa, 1998.

CORASSA, J. de N; CASTELO, P. A. R; STANGERLIN, D. M; MAGISTRALI, I. C. Durabilidade natural da madeira de quatro espécies florestais em ensaios de deterioração em campo. Ciência da Madeira. Pelotas, v. 04, n. 01, p. 108-117, 2013.

CORASSA, J. de N; PIRES, E. M; NETO, V. R. A; TARIGA, T. C. Térmitas associados a degradação de cinco espécies florestais em campo de apodrecimento. Floresta e Ambiente. V. 21 (1), p. 78-84, 2014.

DIAS, F. M.; LAHR, F. A. R. Estimativa de propriedades de resistência e rigidez da madeira através da densidade aparente. Scientia Forestalis. n. 65, p. 102-113, 2004.

GOMES, J. I.; BANDEIRA, A. G. Durabilidade natural de madeiras amazônicas em contato com o solo. Pesquisa em andamento, $\mathrm{n}^{\mathrm{o}} .111, \mathrm{p} .1-5,1983$. 
IAWA COMMITTEE. List of microscope features for hardwood identification. IAWA Bull. New Ser., n. 10, v.3, 1989.

\section{INSTITUTO BRASILEIRO DE GEOGRAFIA E} ESTATÍSTICA- IBGE. Produção da extração vegetal e silvicultura. Rio de Janeiro, v. 30, p.1-48, 2015.

JATI, S. R.; FEARNSIDE, P. M.; BARBOSA, R. I. Densidade da madeira de árvores em savanas do norte da Amazônia brasileira. Acta Amazônica. V. 44, n. 11, p.79-86, 2014.

KÖPPEN, W.; GEIGER, R. Klimate der Erde. Gotha: Verlag Justus Perthes. Wall-map. 1928.

LEPAGE, E. S. Método padrão sugerido pela IUFRO para ensaios de campo em estacas de madeira. Preservação de Madeiras, n.4, p. 205-216, 1970.

MARCONDES, E.; RIBEIRO, M. A.; STANGERLIN, D. M.; SOUZA, A. P.; MELO, R. R.; GATTO, D. A. Resistência natural da madeira de duas espécies amazônicas em ensaios de deterioração de campo. Scientia Plena. V. 9, n. 06, p. 1-9, 2013.

MELO, R. R.; STANGERLIN, D. M.; SANTINI, E. J.; HASELEIN, C. R.; GATTO, D. A.; SUSIN, F. Durabilidade natural da madeira de três espécies florestais em ensaios de campo. Ciência Florestal. Santa Maria. V. 20, n.2, p. 357-365, 2010.

NAHUZ, A. R. Catálogo de madeiras brasileiras para a construção civil. São Paulo, IPT. 2013.

ORGANIZACIÓN INTERNACIONAL DE LAS MADERAS TROPICALES - OIMT. Reseña anual y evaluación de la situación mundial de las maderas 2013-2014, Yokohama, Japón: OIMT. 2014, 223. p.

PILOCELLI, A.; STANGERLIN, D. M.; PEREIRA, R. L.; GATTO, D. A.; CALEGARI, L.; PARIZ, E.; SUSIN, F. Resistencia natural das madeiras de cumaru, cedrinho e paricá submetidas a ensaios de deterioração de campo. Ciência da Madeira. v.6 (1), p. 1-10, 2015.

R Core Team (2016), R: A language and environment for statistical computing. $\mathrm{R}$ Foundation for Statistical Computing, Vienna, Austria. URL https://www.Rproject.org/.

REIS, A. R. S.; ALVES, R. S. dos; URBINATI, C. V.; SANTOS, J . X. dos; SILVA, J. R. da; LISBOA, P. L. B. Anatomia do xilema secundário de sete espécies de Vochysia Aubl.
(Vochysiaceae), conhecidas como quaruba no Estado do Pará, Brasil. Biota Amazônica, V. 5, n. 2, p. 45-51, 2015.

RIBEIRO, M. A.; STANGERLIN, D. M.; SOUZA, A. P.; CARDOSO, G. V.; CALEGARI, L.; GATTO, D. A. Durabilidade natural da madeira de jequitibá em ensaios de deterioração em campo aberto e floresta durante as estações de seca e chuva. Comunicata Scientiae. V. 5, n. 4, p. 402-411, 2014.

SILVA, C. J. da; VALE, A. T. do; MIGUEL, E. P. Densidade básica da madeira de espécies arbóreas de cerradão no estado do Tocantins. Pesquisa Florestal Brasileira. V. 35, n. 82, p. 63-75, 2015

SILVA, M. G.; MORI, F. A.; FERREIRA, G. C.; RIBEIRO, A. O.; CARVAlHO, A. G.; BARBOSA, A. C. M. C. Estudo anatômico e físico da madeira de Schizolobium parahyba var. amazonicum proveniente de povoamentos nativos da Amazônia oriental. Scientia Forestalis, v. 44, n. 110, p. 293$301,2016$.

STANGERLIN, D. M.; CORASSA, J. N.; GATTO, D. A.; PEREIRA, R. L.; CASTELO, P. A. R. Caracterização mecânica de madeiras deterioradas em campo por meio de ultrassom e flexão estática. Comunicata Scientiae. V. 6, n. 3, p. 365-372, 2015.

TREVISAN, H.; MARQUES, F. M. T.; CARVALHO A. G. Degradação natural de toras de cinco espécies florestais em dois ambientes. Floresta. Curitiba, PR, v. 38, n. 1, 2008.

TREVISAN, H.; TIEPPO, F. M. M.; CARVALHO, A. G.; LELIS, R. C. C. Avaliação de propriedades físicas e mecânicas da madeira de cinco espécies florestais em função da deterioração em dois ambientes. Revista Árvore. ViçosaMG, v. 31, n. 1, p. 93-101, 2007.

VALE, A. T.; CARDOSO, T. B.; PEREIRA, R. S.; MARTINS, I. S. Influência das propriedades da casca de Vochysia thyrsoidea na transferência de calor para o câmbio. Revista Eletrônica de Engenharia Florestal. Ano VIII, n. 13, p. 1-12, 2009.

VALE, A. T.; PIMENTEL, J. W.; MENEZZI, C. H.S. D.; DANTAS, V. F. S. Propriedades físicas e mecânicas de sucupira branca Pterodon pubescens (BENTH.) BENTH. Revista Cientifica Eletrônica de Engenharia Florestal. Garça-SP, Ano IX, v. 18, n. 1, p. 118-125, 2011.

ZENID, G. J. Madeira: uso sustentável na construção civil. São Paulo: IPT,2. ed., p. 1-99, 2009. 